Wissenschaften neu zu umgrenzen ${ }^{1}$ ), persönlich aufs wärmste begrüßt, wird es anderen Fachgenossen ${ }^{2}$ ), nicht verargen dürfen, wenn sie sich angesichts mancher dilettantischer Versuche, die gerade in unserer Literatur vorliegen, gegen jeden Einbruch der Philosophie in die Psychiatrie grundsätzlich und mit Entschiedenheit wehren. In keinem Falle aber werden wir kritizistischen Ubungen dieser Art erlauben wollen, uns hinsichtlich unserer praktischen Arbeit Vorschriften zu machen. Wir werden ruhig das Neue betreiben, ohne das Alte zu verketzern und abzuschwören. Wir werden das Wesen und die Erscheinungen der Geisteskrankheiten von allen Seiten, von der anatomischen, neurologischen und serologischen sowohl wie von der psychologischen zu studieren versuchen, und wir werden uns überdies bei jedem Spezialgebiet, das wir selbst wählen, bewußt bleiben, daß seine theoretischen Voraussetzungen, so wie sie uns richtig erscheinen, sicher nicht die sein werden, die eine künftige Generation anerkennen und übernehmen wird.

\title{
ORIGINALIEN.
}

\section{DIE NATÜRLICHEN ABWEHRMITTEL DES KÖRPERS GEGEN DIE SYPHILITISCHE INFEKTION UND IHRE BEEINFLUSSUNG BESONDERS DURCH QUECKSILBER.}

Von

\author{
Dr. S. BERGEL, Berlin.
}

In früheren Arbeiten $^{2}$ ) habe ich gezeigt, daß die syphilitischen Erscheinungen, die anatomisch sich im wesentlichen als entzündliche lymphocytäre Infiltrationen präsentieren, eine nach chemotaktischen Gesetzen sich vollziehende Folge der Spirochäteninfektion, und somit zwar ein Krankheitsprodukt sind, aber gleichzeitig eine natürliche, wenn auch oft unzureichende Abwehrreaktion des Organismus darstellen, die chemisch auf einem Abbau der lipoiden Spirochäten durch die spezifisch eingestellten lipolytischen Lymphocyten beruht, und die serologisch ihren Ausdruck in dem Vorhandensein einer positiven Wassermannschen Reaktion findet. Infolge dieser neugewonnenen Erkenntnisse war überhaupt erst die Möglichkeit gegeben und eine Grundlage geschaffen, auf der man experimentell und klinisch die Frage beantworten konnte, ob ein Heilmittel die natürlichen Abwehrvorgänge des Organismus unterstützt oder verhindert; man wußte eben vorher nicht, selbst wenn man schon annehmen wollte, daß jede „Entzündung" auf alle Krankheitsprozesse irgendwie heilend wirke, was in dieser Allgemeinheit nicht richtig ist, welcher Bestandteil des Entzündungskomplexes gerade bei der Lues das wesentlich heilsame Agens darstelle, aus welchem biologischen Grunde er das sei, und ob, bezw, in welchen Beziehungen diese Vorgänge zur Wassermannschen Reaktion stehen.

LESSER $^{4}$ ) hat nun gesagt, daß das Quecksilber ein Mittel seí, das dem natürlichen Heilungsprozeß entgegenarbeite; das lediglich die Krankheitssymptome beseitige, ohne die Spirochäten zu beeinflussen, und glaubt dafür klinische Belege beigebracht zu haberi. So gewichtig manche auch sein mögen, z. B. die sehr seltenen Reinfektionen nach Quecksilberbehandlung im Gegensatz zu denen nach Salvarsan, so sind doch andere nicht als ${ }^{2}$ wingend und eindeutig anzuerkennen. Vom rein klinischen Standpunkte aus $\mathrm{zu}$ dieser Frage entscheidend Stellung zu nehmen;; steht mir nicht zu, und dürfte auch kaum zu einem Resultate führen, wie die divergierenden Ansichten unter den Klinikern früher und jetzt beweisen.

Aber auf Grund unserer neuen Anschauungen, die uns die biologische Bedeutung der klinischen, Erscheinungen, sowie der anatomischen und serologischen Befunde bei der Syphilis so ungezwuingen und überraschend einfach dem Verständnis nähergebracht haben, will ich versuchen, zur Klärung dieser praktisch sehr wichtigen und wissenschaftlich interessanten therapeutischen: Frage einen Beitrag zu liefern.

Ein gesicherter Beweis, wie das Quecksilber bei der Syphilis wirkt, liegt bisher nicht vor; feststehend ist aber die

\footnotetext{
1) Vergl. A. HOPPE und namentlich R, HOENIGSWALD.

2) Vergl. BLEULER und STRANSKY.

3) Münch. med. Wochenschr. I909 Nr. 2; I9ro Nr. 32; Igr2 Nr. 20; I92I Nr. 36; und Die Lymphocytose Berlin r 921 bei Julius Springer.

7) Dtsch. med. Wochenschr. Ig2I Nr. 2-3; Berl. klin. Wochenschr. I92I Nr. 46.
}

Tatsache, daß es einen Einfluß hat, wahrscheinlich auch auf den Krankheitsverlauf, zum mindesten aber auf die klinisch sichtbaren syphilitischen Krankheitserscheinungen. Wo setzt nun diese Wirkung ein, und wie ist sie zu erklären?

Nach den experimentellen Untersuchungen und klinischen Erfahrungen erscheint es sehr zweifelhaft, wenn nicht ausgeschlossen, daß das Quecksilber in der gebräuchlichen Dosierung die Syphiliserreger unmittelbar und vollständig abtötet, da die Spirochäten tötende Dosis bereits eine für den menschlichen Organismus giftige ist. Es ist also anzunehmen, daß sein Einflu $\beta$, falls er überhaupt, wenn auch unzureichend, sich auf die Spirochäten erstrecken sollte, über die natürlichen Mittel des Körpers geht, oder daß er ein ausschließlich symptomatischer, lediglich die sichtbaren Krankheitsprodukte betreffender ist. In beiden Fällen erhebt sich die Unterfrage, welcher Art dieser Einfluß ist.

In dieser Hinsicht erscheint es von Wichtigkeit, daß eine spezielle Einwirkung des Quecksilbers auf lymphoides Gewebe anscheinend sichergestellt ist. Nachdem wir, wie gesagt, jetzt wissen, daß die Lymphocyten und ihre Bildungsorgane, die Lymphdrüsen, die Antistoffbildner gegenüber den Spirochäten sind, daß diese Schutz- und Heilwirkung auf einer spezifischen Lipasenwirkung gegenüber dem Lueslipoid beruht, deren biologischer Ausdruck die Wassermannsche Reaktion ist, ist vielleicht gerade in den Beziehungen des Quecksilbers zu dem für die syphilitischen Krankheitsprodukte so charakteristischen lymphoiden Gewebe der Schlüssel zur Lösung der Frage nach seiner Wirkung überhaupt zu finden.

Am besten läßt sich der Einfluß der Quecksilberbehandlung anatomisch und klinisch beim Primäraffekt verfolgen, und hier werden wir auch am ehesten imstande sein, eine biologische Deutung des Heilprozesses zu geben.

LESSER selbst kennzeichnet den Unterschied zwischen der Salvarsan- und der Queckilberwirkung beim Primäraffekt nach den Untersuchungen von STEJSKAI dadurch, daß nach Salvarsaninjektionen die chemotherapeutisch getroffenen Spirochäten plötzlich verschwinden, und daß das Infiltrat sich allmählich zurückbildet, während nach Quecksilbereinwirkung allmähliches Schwinden der Spirochäten aus dem Primäraffekt entsprechend der Rückbildung des Infiltrates erfolgt.

Wenn wir diesen Befund, und ähnlich dürfte er auch bei anderen syphilitischen Manifestationen sein, von unserem Gesichtspunkte aus biologisch analysieren, so ergibt sich ungezwungen folgende Erklärung. Die Spirochäten werden durch das Salvarsan direkt primär abgetötet, ihre z.T. lipoiden Zerfallsprodukte werden von den dadurch vielleicht noch vermehrten Reaktionszellen, den lipolytischen Lymphocyten, aufgenommen bzw. weiter abgebaut. Wenn sie diese Funktion erfüllt haben, und wenn Spirochäten nicht mehr vorhanden sind, verschwinden sie naturgemäß, da die Ursache ihres Auftretens beseitigt ist. Das Quecksilber dagegen tötet die Spirochäten nicht unmittelbar ab; daher kommt es auch nicht zu einem plötzlichen Verschwinden derselben, sondern das Quecksilber wirkt hier auf die lymphoide Umwallungszone, die der Körper als Abwehrreaktion gegen die Spirochäten aufgerichtet hat, und zwar, nach den anatomischen, serologischen und klinischen Befunden zu urteilen, derart, daß immer ein Teil der Lymphocyten zerfällt, was zur Folge hat, daß einerseits das Infiltrat allmählich kleiner wird, und daß 
andererseits durch die jedesmal aus den Lymphocyten freiwerdende Lipase ein Teil der lipoiden Spirochäten abgetötet bzw. abgebaut wird. Durch den allmählichen Zerfall der Lymphocyten, d. h. bei der Rückbildung des Primäraffektes und ebenso der anderen syphilitischen Erscheinungen wird also nicht bloß das Krankheitsprodukt symptomatisch beeinfluBt, sondern es findet indirekt auch eine Einwirkung auf die Spirochäten selbst statt, sie werden allmählich weniger, verschwinden aber meist nicht gänzlich. Denn die Wirkung auf die Spirochäten hält eben nur so lange an, als Lymphocytenlipase vorhanden ist, die die lipoiden Erreger abbauen kann. Während also beim Salvarsan primär die Spirochäten getötet werden, und sekundär die Folgeerscheinung, der lymphocytenreiche Primäraffekt, sich zurückbildet, schwinden nach Quecksilberwirkung die Spirochäten zu einem Teile erst sekundär dadurch, daß sie auf dem Umwege über den durch das $\mathrm{Hg}$ erzeugten Zerfall der Lymphocyten und die hierbei freigewordene Lipase stark verringert, aber meist nicht in ihrer Gesamtheit abgebaut werden. Während so die entzündlichen Erscheinungen schwinden, der sichtbare Krankheitsprozeß also eine wesentliche Besserung zeigt, unter Umständen sogar eine Heilung vortäuschen kann, ist in Wirklichkeit nur ein Zustand von latenter, wenn auch vielleicht geringerer Infektion vorhanden, bzw. künstlich erzeugt. Daher kommt es auch, daß eine abortive Heilung nach Quecksilber so selten gelingt, daß spätere Erscheinungen nicht ausbleiben.

Mit dieser Erklärung der Quecksilberwirkung bei allen syphilitischen Manifestationen würde auch die Tatsache übereinstimmen, daß im Beginn der Behandlung eine negative WaR., eben infolge des Freiwerdens der Lipasen, zunächst oft positiv, und eine schwache Reaktion oft in eine stärker positive umgewandelt wird, um mit dem Schwinden der Lymphocytenlipasen allmählich wieder negativ zu werden. Meist parallel mit dem Auftreten der WaR. geht das Aufflammen der entzündlichen Erscheinungen am Körper auch nach Quecksilberbehandlung, die HerXHEIMER sche Reaktion. Wäre es richtig, was LESSER sagt, daß das Quecksilber lediglich die Krankheitssymptome beseitigt und den Abwehrkräften des Körpers direkt entgegenarbeitet, so müßten beim Primäraffekt die lymphocytären Infiltrate, die ja im wesentlichen die natürlichen Heilmittel darstellen, ohne ihre Wirkung auszuüben, einfach verschwinden, abwandern, oder ihre wirksame Substanz müßte durch das Quecksilber zerstört werden. Die Folge davon müßte notwendigerwcise die sein, daß zwar der Primäraffekt kleiner wird, daß aber die Spirochäten, da jetzt der Widerstand von seiten des Körpers wegfällt, nicht bloß nicht zugrunde gehen, sondern sich in dem für sie geeigneten Medium ungehindert vermehren. Die experimentellen Untersuchungen, die LESSER selbst anführt, ergeben aber im Gegensatz hierzu, daß mit der Rückbildung des Infiltrates die Spirochäten allmählich verschwinden, $z$. T. wenigstens abgetötet werden. Das läßt sich nur so erklären; daß infolge des Lymphocytenzerfalles (Zelltrümmer und Kernreste werden bei der mikroskopischen Untersuchung der Primäraffekte auch tatsächlich gefunden) das Infiltrat sich zurückbildet, kleiner wird, und daß durch die freigewordene Lipase die lipoiden Spirochäten mehr oder weniger abgebaut werden. Die Annahme, daß die Spirochäten primär durch das Quecksilber abgetötet werden könnten, findet, wie gesagt, weder in den Experimentaluntersuchungen noch in dem klinischen Ablauf des Heilungsprozesses eine Stütze; denn in diesem Falle würden ja zuerst die Spirochäten schwinden und dann das Infiltrat sekundär sich zurückbilden. So wie beim Primäraffekt dürften die Verhältnisse sich auch bei den übrigen syphilitischen Manifestationen abspielen. Auch das Zustandekommen der HerXHEIMER schen Reaktion und das häufige Positivwerden eines negativen Wassermann im Beginn der Quecksilberbehandlung dürfte LESSER nach seiner Theorie wohl kaum erklären können, während sich nach unserer Anschauung beides von selbst ergibt.

Auf den Umstand, daß wahrscheinlich beim Quecksilber ebenso wie bei anderen differenten Mitteln die Dosierung eine nicht unwesentliche Rolle spielt, indem kleine Dosen anregend, reizend, fördernd, starke dagegen lähmend und abtötend wirken, will ich hier nicht eingehen.

Besteht aber diese Art der Quecksilberwirkung zu Recht, wofür klinische und biologische Tatsachen sprechen, dann kann man nicht ohne weiteres behaupten, daß das Quecksilber nur die Symptome beseitigt und den natürlichen $\mathrm{Ab}$ wehrkräften direkt entgegenarbeitet. LESSER hätte nach meinen Anschauungen dann recht, wenn man beweisen könnte, đaß das Quecksilber entweder die natürlichen Abwehrzellen, die Lymphocyten, einfach zur Abwanderung bringt, die lymphocytäre Infiltration restlos beseitigt, ohne daß sie vorher ihre biologischen Wirkungen $\mathrm{zu}$ entfalten vermag, oder, $\mathrm{da} B$ es die wirksame Lipase der lymphocytären Elemente zerstört. Dieser Beweis ist aber bisher nicht erbracht, und es wäre auch biologisch schwer verständlich und ohne Analogien, wollte man annehmen, daß die syphilitischen Manifestationen auf Quecksilber dadurch zurückgehen, daß die Lymphocytenherde einfach als unversehrte Zellen resorbiert werden und verschwinden, und daß auf diese Weise nicht bloß die Rückbildung des Primäraffektes und der übrigen klinischen Erscheinungen, sondern auch die Verkleinerung der Lymphdrüsen zustandekommt.

Das Quecksilber wirkt also, und darin hat LESSER wohl recht, primär auf die Krankhejtsprodukte, im wesentlichen auf die lymphocytären Infiltrate, aber aus den angeführten Gründen ist deswegen seine Wirkung noch nicht eine nur symptomatische und direkt entgegengesetzt den natürlichen Abwehrkräften; die zur Zeit vorhandenen werden sogar wahrscheinlich akut zur Entfaltung gebracht. Insofern allerdings hindert das Quecksilber, besonders bei zu langer Anwendung, auch nach unserer Anschauung zwar nicht die akute, wohl aber die anhaltende, dauernde, kontinuierliche $\mathrm{Ab}$ wehrwirkung der lebenden lymphocytäiren Reaktionszellen, als nach ihrem Zerfall nur die gerade im Zellleibe vorhandene Lipase frei und wirksam wird, aber die ständige Produktion von Antistoffen aufhört, und zunächst kein Neuersatz eintritt. Das hat zur Folge, daß zwar ein Teil der Spirochäten abgetötet wird, da $\beta$ vor allem die sichtbaren entzündlichen Erscheinungen schwinden und damit die WaR. allmählich negativ wird, daß aber dann die übriggebliebenen Spirochäten sich ungehindert vermehren können und wieder von frischem Lymphocyteninfiltrate als Abwehrreaktionen hervorrufen, daß also über kurz oder lang klinisch Rezidive mit positivem Wassermann auftreten. Dadurch kann zunächst klinisch eine Heilung vorgetäuscht werden, indem die Manifestationen und mit ihnen die WaR. schwinden, aber nur sehr selten werden sämtliche Spirochäten abgetötet, so daß es, und das ist unter diesen Umständen noch der biologisch günstigere Verlauf im Vergleich zu den sog. 1atenten Fällen, nicht aus. bleibt, daß die überlebenden Spirochäten von neuem lymphocytäre entzündliche Reaktionen mit positivem Wassermann, klinisch also Rezidive, bedingen.

Da das Quecksilber also durch Entbindung der wirksamen Substanz der vorhandenen Abwehrzellen zwar die klinischen Erscheinungen beseitigt und auch die Abtötung eines Teiles der Spirochäten fördert, dafür aber gewaltsam die Dauerwirkung der sonst ständig. Antistoffe produzierenden Zellen durch ihren Zerfall für einige Zeit unterdrückt, muß man sich dieser unzureichenden Wirkung bewuBt sein und sich durch Scheinheilungen nicht täuschen lassen. Aus dem rezidivierenden Verlauf der unbehandelten Syphilis einerseits und der geschilderten eigentümlichen Wirkungsweise des Quecksilbers auf der anderen Seite ergab sich schon von selbst das klinische Bedürfnis nach wiederholten Quecksilberkuren, die aber nach unseren Darlegungen nur dann biologisch einen Sinn, und daher therapeutisch nur dann einen Erfolg haben können, wenn entweder manifeste Erscheinungen vorliegen, oder ein positiver Wassermann in der Latenzperiode vorhanden ist, der darauf schließen läßt, daß im Innern des Körpers lymphocytäre Herde sich befinden, durch deren Lipasen die Spirochäten beeinflußt werden könnten. Viele klinische Erfahrungen, auf die ich hier im einzelnen nicht eingehen kann, sprechen durchaus in diesem Sinne. Ganz in Ubereinstimmung damit hat aus großer klinischer Er- 
fahrung heraus erst jüngst wieder, wie vor ihm schon andere, ein so hervorragender Kenner der Syphilis, wie Blaschko ${ }^{1}$, darauf aufmerksam gemacht, erstens, daß es unzweckmäßig sei, die Behandlung mit Quecksilber zu frühzeitig, d. h. wenn die Reaktionserscheinungen des Körpers sich noch nicht völlig ausgebildet haben, $z \mathfrak{u}$ beginnen, und zweitens, da $B$ es nicht rationell sei, ,bei fehlenden Krankheitserscheinungen und negativem Wassermann eine prophylaktische Behandlung einzuleiten". Wenn man nämlich stets sofort nach dem Auftreten schon der ersten geringen entzündlichen Erscheinungen, wo also nach unserer Anschauung noch wenig lymphocytäre Abwehrherde vorhanden sind, mit der Quecksilberbehandlung beginnt, so erstickt man ihre weitere Ausbildung und Auswirkung im Keime, und schafft für die Queckșilberbehandlung insofern ein nur geringes Wirkungsfeld, als man dann nur eine geringe Ausbeute an wirksamen Stoffen erhält, so daß nur ein kleiner Teil von Spirochäten zerstört wird, während bei starken, ausgebreiteten, einige Zeit bestehenden Symptomen, wo also reichliche Abwehrelemente im Organismus vorhanden sind, der Zerfall dieser Zellmassen eine große Menge wirksamer Lymphocytenlipasen für den Spirochätenabbau frei werden läßt. Bei negativer WaR. und fehlenden Erscheinungen muß überhaupt jeder Erfolg ausbleiben, weil ein Angriffspunkt für die Quecksilberwirkung gar nicht vorhanden ist, und oft tritt auch schon unmittelbar nach der Behandlung ein Exanthem auf. Es besteht aber selbst bei starken Manifestationen immer die Gefahr, daß nach dem Abklingen der an sich schon oft unzureichenden Lymphocytenwirkung ohne frischen Nachschub die übriggebliebenen vollentwickelten oder nur teilweise abgebauten Spirochäten früher oder später neue Krankheitserscheinungen hervorrufen. Daher ist die kausale Behandlung der Syphilis durch Salvarsan der alleinigen . Quecksilberbehandlung prinzipiell vorzuziehen, solange wir noch keine spezifische immunbiologische besitzen.

Es erscheint indessen nach unseren Auseinandersetzungen von den verschiedenen Angriffspunkten und der verschiedenen Wirkungsart des Salvarsan und des Quecksilbers zweckmäßig und rationell, was die Klinik schon erfahrungsgemäß bestätigt hat, in gewissen Fällen eine kombinierte Behandlung, allerdings in bestimmter, biologisch begründeter Reihenfolge anzuwenden.

Nicht bloß beim Primäraffekt, sondern auch bei den syphilitischen Manifestationen späterer Stadien muß es leichter möglich sein, eine radikale Heilung des ganzen Prozesses zu erzielen, wenn an eine kräftige Salvarsantherapie, durch die, wennschon nicht alle, so doch der größte Teil der Spirochäten vernichtet ist, dort, wo noch positiver Wassermann vorhanden ist, eine leichte Quecksilberbehandlung sich anschließt, um dadurch diejenigen Spirochäten, die möglicherweise durch das Salvarsan nicht getroffen sind, durch die die Spirochätenherde einkreisenden Lymphocyteninfiltrationen, bzw. ihre in Freiheit gesetzten Lipasen noch nachträglich und auf anderem Wege abzutöten. Vielleicht gelingt es auf diese Art, auch arsenresistente Spirochäten auf dem Umwege über das Quecksilber durch die eigenen Abwehrkräfte des Körpers abzubauen. Die Knetmassage des Primäraffektes, die LESSER nach Salvarsaninjektionen empfiehlt, beruht vielleicht auch darauf, daß die lymphocytären Zellen zerquetscht, und ibre Lipasen in Freiheit gesetzt werden.

Es wird uns so einleuchtend, warum es zweckmäßig und notwendig ist, erst das Salvarsan, das unmittelbar auf die Spirochäten wirkt, anzuwenden, und dann erst das Quecksilber, das die durch das Salvarsan noch in vermehrter Menge an den Krankheitsherd angelockten lymphocytären Reaktionszellen beeinflußt, einwirken zu lassen. Es wird uns aber auch biologisch verständlich, warum in der Latenz, bei fehlenden Erscheinungen und negativem Wassermann zwar eine Quecksilberbehandlung nutzlos ist, was klinisch auch bestätigt wird, weil eben der Angriffspunkt für die $\mathrm{Hg}$-Therapie fehlt, warum aber auch bei diesen Fällen latenter Syphilis mit negativem Wassermann eine Salvarsanbehandlung durchaus noch von Nutzen sein kann, weil wị wịssen, daß die negative Berl, klin. Wochenschr, xg2r Nr, 4I,
WaR., die ja nur das Fehlen von entzündlichen lymphocytären Infiltrationen anzeigt, das Vorhandensein von Spirochäten im Körper nicht ausschließt, die durch das Salvarsan immer noch getroffen werden können.

Hinzu kommt noch der Umstand, daß nach Salvarsaninjektionen außer der rein spirilloziden Wirkung eine Lymphocytose entsteht, daß um Salvarsankörner Lymphocyten sich ansammeln, und da $B$ während bezw. infolge der Salvarsanbehandlung in die syphilitischen Herde größere Mengen von Lymphocyten und deren Abkömmlingen, Plasmazellen, angelockt werden, so daß man pathologisch-anatomisch bei der Rückbildung der Hautsyphilide, bei der Besserung der klinischen Erscheinungen eine Zunahme der Lymphocyten im Krankheitsherde konstatieren kann, was mit den Heilungsvorgängen sicher im Zusammenhang steht.

Die Lymphocytose anregende Wirkung des Salvarsan würde auch neben der durch den Spirochätenzerfall reaktiv hervorgerufenen Lymphocytose das Positivwerden einer negativen WaR. nach provokatorischen Einspritzungen erklären.

Bemerkenswert ist es, daß auch das Jod, das bei der Syphilis so günstige Heilwirkungen ausübt, eine ausgesprochene Lymphocytose erzeugt. Das Pilocarpin, das früher oft und angeblich erfolgreich angewandt wurde, ruft ebenfalls Lymphocytose hervor.

Es ist jedoch auch möglich, daß das Quecksilber, das ja im wesentlichen als Sublimat im Körper zur Wirkung gelangt, noch auf eine ganz andere Art einen Einfluß ausübt, sofern man nicht überhaupt eine mehrfache, verschiedene Angriffs- und Wirkungsweise desselben voraussetzen will.

Es ist bekannt, daß das Lecithin adsorbierende, giftbindende Eigenschaften gegenüber dem Sublimat besitzt. $\mathrm{Da}$ wir gesehen haben, $\mathrm{da} B$ das syphilitische Antigen chemisch eine besondere Ähnlichkeit mit dem Lecithin hat, so kann man sich vorstellen, daß auch im Organismus das Sublimat elektiv von denjenigen Stellen gebunden, adsorbiert wird, wo sich das diesem Lecithin ähnliche luetische Lipoid befindet. Während dieses Adsorptionszustandes ist das lipoide Antigen zeitweise gewissermaßen außer Aktion gesetzt, wenn auch nicht dauernd unwirksam gemacht, die reaktive Lymphocytenansammlung wird, zunächst wenigstens, verschwinden, mit ihr auch die klinischen Erscheinungen, und die WaR. wird negativ werden. Wird aus irgendeinem Grunde das Lipoid wieder frei, so kommt es, wenn die antigene Kraft desselben nicht gebrochen ist, von neuem zu entzündlicher Reaktion, der Wassermann wird positiv. So würde sich die Tatsache erklären lassen, daß man imstande ist, in vitro durch Hinzufügen ganz geringer Mengen Sublimats zu wassermannpositiven Seren Syphilitischer die Reaktion negativ werden zu lassen, indem das zum Zustandekommen der Reaktion notwendige, dem Lecithin chemisch ähnliche Iuetische Lipoid durch das zugesetzte Sublimat adsorbiert wird, und infolgedessen nicht mehr mit der Lipase des Serums in Verbindung treten kann. In gleichem Sinne sind vielleicht auch die Versuche von v. WASSERMANNs zu deuten, der durch Quecksilbereinreibung die positive Reaktion nichtsyphilitischer Kaninchenseren in eine negative umwandeln konnte. Hier kann es sich ja gar nicht um eine Einwirkung auf die Syphilisspirochäten handeln, sondern hier spielt wahrscheinlich die Adsorption des Quecksilbers durch das dem Kaninchen gerade eigentümliche, dem Iuetischen ähnliche Lipoid eine Rolle.

Eingehende experimentelle Untersuchungen über die pharmakologische Wirkungsweise des Quecksilbers unter Berücksichtigung der geschilderten biologisch-klinischen Gesichtspunkte sind jedenfalls dringend notwendig.

Auf Grund unserer biologischen Anschauungen, die mit den anatomischen Befunden und klinischen Erfahrungen übereinstimmen, müssen wir sagen, daß das Quecksilber die jeweilig vorhandenen Abwehrkräfte des Organismus zu einer einmaligen, meist unzureichenden Auswirkung auf die Spirochäten gelangen läßt, aber die dauernden natürlichen Heilungsvorgänge durch Vernichtung der lebenden Produktionsquellen der Antistoffe zeitweise verhindert, ohne selbst unmittelbar therapeutisch auf die Krankheitserreger einzu. wirken. 
Der alte und immer von neuem sich geltend machende Gegensatz zwischen Merkurialisten und Antimerkurialisten wird sich infolge der vertieften biologischen Einsicht in die feineren Vorgänge bei dem natürlichen Heilproze $B$ und der Quecksilberwirkung wahrscheinlich in ähnlicher Weise ausgleichen, wie der zwischen den Ärzten, welche die Entzündung mit Eis, und denen, die sie mit Staunng behandeln. Beide glauben damit gute Erfolge gesehen zu haben. Die Beobachtung der grobsinnlich wahrnehmbaren klinischen Resultate war richtig, aber die Deutung der Beobachtung, der Wirkung des Heilmittels war falsch. Während das Eis die an sich heilsame Wirkung der ,Entzündung" zunächst fast lähmt, um sie dann durch die reflektorisch folgende Kongestion stärker anzufachen, zu fördern, ist es beim Quecksilber umgekehrt; zuerst tritt auf dem Umwege über die Abwehrmittel des Körpers eine Verausgabung der vorhandenen Antistoffe und damiteine gewisse, wenn auch unzureichende Heilwirkung ein, mit der Erschöpfung dieser Mittel schwinden zwar naturgemäß die klinischen Symptome, aber, und das ist das Unzweckmäßige, die Quelle für die weitere dauernde Antistoffbildung wird für eine Zeit verstopft, ohne daß das Mittel selbst die Spirochäten direkt abtötet, so daß als natürliche, heilsame Reaktion des Körpers Rezidive auftreten.

\section{ÜBER SALVARSAN-HIRNTOD'1).}

Von

\section{Prof. R. Henneberg, Berlin.}

Die nachstehenden Mitteilungen vermehren die veröffentlichte Kasuistik der Salvarsantodesfälle um 3 weitere Beobachtungen. Es handelt sich durchweg um Fälle von tötlicher Salvarsanwirkung auf Grund eines im Gehirn lokalisierten Vorganges. Die Gründe, die mich veranlassen, die üblichen Bezeichnung "hämorrhagische Salvarsan-Encephalitis" zu. vermeiden, ergeben sich aus der Arbeit. Die außerordentliche praktische Bedeutung der den Salvarsanhirntod betreffenden Fragen und der Umstand, daß wir zurzeit noch weit davon entfernt sind, die Bedingungen, unter denen er in die Erscheinung tritt; zu übersehen, läßt die Veröffentlichung eines jeden genauer beobachteten Falles angezeigt erscheinen. In folgenden 3 Fällen konnte ich eine eingehende Untersuchung des Gehirns vornehmen.

Fall I. M. M., Arbeiterin, 3I Jahre alt. I. Aufnahme in ein städtisches Krankenhaus 20. III. 1918. Seit Mai 1917 heftige Kopfschmerzen und Schwindelanfälle, Behandlung mit Calomel, Salvarsan und Schmierkur bis Januar 1918. Befund bei der Aufnahme: L. Pupille leicht entzündet, Lichtreaktion prompt, Gehirnnerven frei, Gaumenbögen gerötet, systolisches Geräusch on der Basis, Milz anscheinend vergrößert, Reflexe lebhaft, Na-kensteifigkeit geringen Grades, starker Kopfschmerz und Schwindel Lumbalpunktatleicht getrübt, viel Lymphocyten, Albumosen : leichte Trübung, Erhöhung des Druckes (ca. 300). In der Folge Kopfschmerzen und Nackensteifigkeit wechselnden Grades, Kopf klopfempfindlich, Wassermann positiv. Behandlung: Inunktionskur, dann Neosalvarsan, im ganzen 0,9 und einige Hg-Spritzen. 3I. 5. I9i8 weitgehend gebessert entlassen.

4. IO. I9I8 2. Anfnahme. In der Zwischenzeit zeitweilig Kopfschmerzen. Seit 3 Wochen Doppeltsehen, Wassermann August I9r8 positiv, 1. Pupille entrundet, Lichtreaktion erhalten. Inunktionskur: 6 Touren, Neosalvarsan: 5 Dosen, im ganzen I,4 g. Beschwerdefrei bis auf Doppelbilder entlassen.

I3. II. I9I9 3. Aufnahme. Seit I4 Tagen Kopf- und Rückenschmerzen, zeitweilig Doppelsehen. Befund: Augenhintergrund normal, Residuen einer Trochlearisparese, Pupillen entrundet, reizfähig, Schwellung der Halsdrüsen, Herztöne rein, 1. hinten unten Dämpfung (Pleuritis). Reflexe lebhaft, sonst regelrecht, Urin frei von Eiweiß. Wassermann positiv. Inunktionskur 6 Touren, Neosalvarsan 4 Dosen, im ganzen 0,9 g. 2. IV. I919 beschwerdefrei entlassen.

8. IX. Igrg 4. Aufnahme. Patientin war in der $Z$ wischenzeit beschwerdefrei. Befund: L. Pupille Spur weiter als die r., beide entrundet, Lichtreaktion prompt, Gehirnnerven frei, Submaxillardrüsen geschwollen, kraterförmiger Defekt in der r. Tcnsille, systolisches Geräusch an der Basis, Reflexe regelrecht, lebhaft. Ab 9. IX. I9I9 Inunktionskur in der gewöhnlichen Form, ab 22. IX.

ग) Vortrag in_der Berl. Ges. f. Psych, u._Nervenkrankh. am 12. VII. Igzo.
1919 Natr. jod. $3: 200,3$ mal täglich I EBlöffel, Neosalvarsan 15. IX. I919: 0, I5; 22. IX. I919:-0;3; 29. IX. I920: 0,3; 6. X. 1919: 0,3; I3. X. 1919: 0,3; 20. IX. 1919: 0,45; darach Brechreiz. 2I. X. IgIg fühit sich Patientin noch etwas schlecht, Hustenreiz, stechende Schmerzen 1. hint. unten. 23. X. 1919 morgens Erbrechen, Temperatur 37,5, beim Aufstehen etwas wirr, zieht sich verkehrt an, taumelt, erkennt die Schwester nicht, befolgt aber Aufforderungen. Eine halbe Stunde später reagiert sie noch auf Anruf, bringt keine Klagen vor, führt aber aufgetragene Bewegungen nicht aus, bald darauf Unruhe, sirebt aus dem Bett, redet unverständlich, eigenartig starre Bewegungen der Arme, dann zunehmende Benommenheit, abends tiefes Koma, Temperatur $38,2, r$. Bulbus im inneren Winkel, Pupillen mittelweit, reagieren, Reflexe an den Armen lebhaft, Bauchreflexe fehlen, starkes Nachröten der Haut, Knie- und Achillesreflex lebhaft, unsicherer Babinski 1. Keine Nackensteifigkeit. 24. X. I9 I9 Temperaturanstieg, morgens 38,3 , abends 39,3 , Cyanose, Trachealrasseln, Schweiß, Spasmen in den Armen, Puls gespannt ca. 120, Aderlaß $160 \mathrm{ccm}$, Kochsalzinjektion intravenös $120 \mathrm{ccm}$, abends Spasmen in Armen und Beinen, Babinski $r$. und 1., Hände in Hyperflexionstellung, starke Cyanose, Atmung langsam, krampfhaft, Puls zeitweilig unregelmäßig, Aderlaß 200, intravenöse Kochsalzinfusion mit Traubenzuckerzusatz, 25. X. I9I9 morgens Temperatur 4I,5, Exitus.

Sektionsbefund: Kleine weibliche Leiche, guter Ernährungszustand, Schädeldach mittelschwer, Dura nicht verwachsen, etwas trocken, weiche Hirnhäute überall zart, durchsichtig, glatt, spiegelnd, feuchț, Gefäße prall gefüllt, Windungen abgeflacht, Gefäße an der Basis ohne krankhaften Befund, Intima glatt.

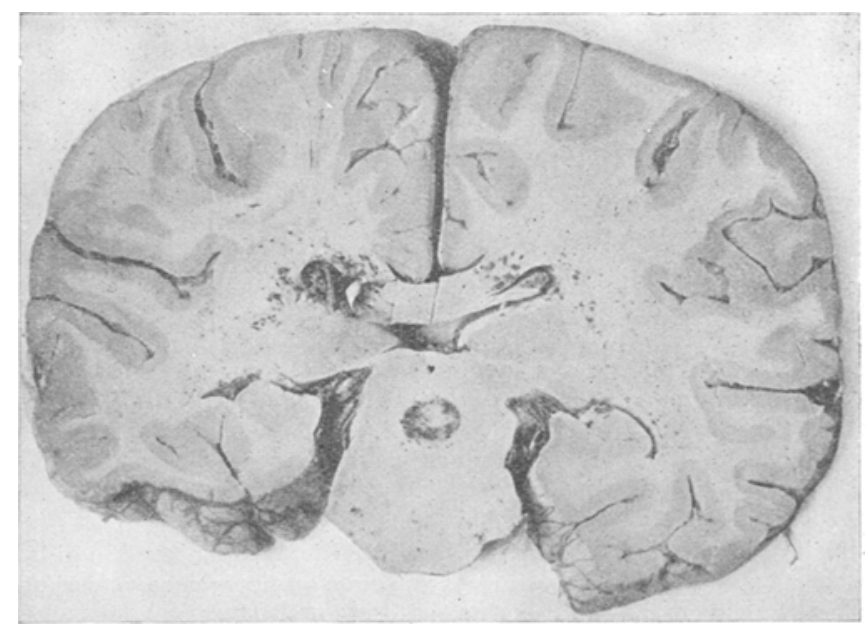

Abb. I.

Fall I. Salvarsanhirnpurpura. Blutungen im Balken, in der Umgebung der Seitenventrikel und in den Bindearmen.

Herzmuskel braunrot, Klappen leicht verdickt, Aorta ohne Veränderungen, 1. Lunge stark verwachsen, beide sehr stark bluthaltig, einzelne luftleere Läppchen mit körniger Schnittfläche, Milz etwas vergrößert, Nieren auch mikroskopisch völlig normal, ebenso die Leber.

Nach Formalinhärtung des Gehirnes zeigen Frontalschnitte: Im Stirnhirn finden sich mäßig zahlreiche Petechien, und zwar ziemlich symmetrisch angeordnet im Balken und im Bereich der inneren Kapsel, und zwar im dorsalen Teil derselben. Rinde, Mark und große Ganglien sind völlig frei. Nach hinten nehmen die Blutungen stark zu. Frontalebenen durch die Mitte des Linsenkernes zeigen in der oberen Hälfte der inneren Kapsel sehr gedrängte Petechien, solche finden sich auch beiderseits im Balken, sie liegen an der Einstrahlungsstelle des Balkens und lassen das Mittelstück frẹi. Wenig zahlreich sind die Petechien in dem Markgebiet $z$ wischen Balken und innerer Kapsel. Schnitte, die durch das Pulvinar fallen (Abb. I) zeigen Petechien in den lateralen Teilen des Balkens, in der nächsten Umgebung des Seitenventrikel und der Unterhörner; kleine Gruppen und einzelne Blutungen finden sich auch in dem den Pulvinar außen anliegenden Hemisphärenmark. Besonders auffâllig ist der Befund einer starken hämorrhagischen Durchsetzung der Bindearmkreuzung, nach hinten läßt sich diese noch eine Strecke weit in den Bindearmen verfolgen. In Schnitten durch den hinteren Teil der Scheitellappen und durch die Hirtarhauptslappen zeigt sich das Splenium fast völlig frei. Die Blutungen finden sich fast ausschließlich in der AuBenwand des Ventrikeis. Die Enden der Hinterhörner sind allseitig von Hämorrhagien umgeben. Uberall ist eine sehr starke Hyperämie 